\title{
EFFECTIVE MAGNETIC FIELDS IN ULTRACOLD ATOMIC GASES *
}

\author{
G. Juzeliūnas ${ }^{\mathrm{a}}$, J. Ruseckas ${ }^{\mathrm{a}}$, and P. Öhberg ${ }^{\mathrm{b}}$ \\ ${ }^{a}$ Institute of Theoretical Physics and Astronomy of Vilnius University, A. Goštauto 12, LT-01108 Vilnius, Lithuania \\ E-mail: gj@itpa.lt \\ ${ }^{\mathrm{b}}$ Department of Physics, University of Strathclyde, Glasgow G4 ONG, Scotland
}

Received 25 April 2005

\begin{abstract}
We consider the influence of two resonant laser beams (to be referred to as the control and probe beams) on the mechanical properties of a degenerate atomic gas. The control and probe beams of light are assumed to have orbital angular momenta (OAM) and act on the three-level atoms in the electromagnetically induced transparency (EIT) configuration. We have carried out an explicit analysis of the quantum dynamics of the atoms coupled with the two laser beams. Using the adiabatic approximation, we have obtained an effective equation of motion for the atoms driven to the dark state. The equation contains a vector potential type interaction as well as an effective trapping potential. The effective magnetic field is shown to be oriented along the propagation direction of the control and probe beams containing OAM. Its spatial profile can be controlled and shaped by choosing the proper laser beams. We have demonstrated how to generate a constant effective magnetic field both in the disc and ring geometries of the atomic trap. We have also studied situations where the effective magnetic field exhibits a radial dependence. We have shown that the effective magnetic field can be concentrated within a region where the effective trapping potential holds the atoms. Furthermore the estimated magnetic length can be considerably smaller than the size of the atomic cloud.
\end{abstract}

Keywords: slow light, electromagnetically induced transparency, ultracold atomic gases, degenerate Fermi gases, atomic Bose-Einstein condensates, effective magnetic field, dark states

PACS: $42.50 . G y$, 42.50.Fx, 03.75.Ss

\section{Introduction}

Recent experimental advances in trapping and cooling atoms have made it possible to produce atomic Bose-Einstein condensates (BECs) [1-4] and degenerate Fermi gases [5-7] forming at temperatures of the millikelvin range. The atomic BECs and degenerate Fermi gases are the systems where an atomic physicist often meets the physical phenomena encountered in condensed matter physics. For instance, atoms in optical lattices are often studied using the Hubbard model [8] familiar from the solid state physics.

Ultracold atomic gases have turned out to be a remarkably good medium for studying a wide range of physical phenomena. This is mainly due to the fact that it is relatively easy to experimentally manipulate parameters of the system, such as the strength of interaction between the atoms, properties of a lattice in which the atoms are trapped, the geometry of an external trap, etc. Such a freedom of manipulating the parameters is

\footnotetext{
* The report presented at the 36th Lithuanian National Physics Conference, 16-18 June 2005, Vilnius, Lithuania.
}

usually not accessible in other systems known from the condensed matter or solid state physics.

Atoms forming quantum gases are electrically neutral particles, and there is no vector potential type coupling of the atoms with a magnetic field. Therefore, a direct analogy between the magnetic properties of degenerate atomic gases and solid state phenomena is not necessarily straightforward. It is possible to produce an effective magnetic field in a cloud of electrically neutral atoms by rotating the system so that a vector potential would appear in the rotating frame of reference [9-11]. This would correspond to a situation where the atoms feel a uniform magnetic field. Yet stirring an ultracold cloud of atoms in a controlled manner is a rather demanding task.

There have also been suggestions to take advantage of a discrete periodic structure of an optical lattice to introduce assymetric atomic transitions between the lattice sites [12-14]. Using this approach one can induce a nonvanishing phase for the atoms moving along a closed path on the lattice, i. e. one can simulate a magnetic flux [12-14]. However, such a way of creating the 
effective magnetic field is inapplicable to an atomic gas that does not constitute a lattice.

A significant experimental advantage would be gained if a more direct way could be used to induce an effective magnetic field. In the previous papers $[15,16]$ we have shown how this can be done using two light beams in an electromagnetically induced transparency (EIT) configuration. Here we present a more detail analysis of the phenomenon for various spatial distributions of the laser fields. We demonstrate that if at least one of these beams contains an orbital angular momentum (OAM), an effective magnetic field appears, which acts on the electrically neutral atoms. In other words, the coupling between the light and the atoms will provide an effective vector potential in the atomic equations of motion. Compared to the rotating atomic gas, where only a constant effective magnetic field is created [9-11], using optical means will be advantageous since the effective magnetic field can now be shaped by choosing the proper control and probe beams. Note that the appearance of the effective vector potential is a manifestation of the Berry connection which is encountered in many different areas of physics [17-19].

The outline of the paper is as follows. In Sect. 2 we present the general theory of adiabatic motion of multilevel atoms. The equations of motion contain the terms due to effective vector and trapping potentials describing an effective magnetic field. In contrast to our previous letter [15], the emerging effective potentials are now fully Hermitian. Yet, the two formulations are equivalent. In Sect. 3 we define a system of three level atoms in the $\Lambda$-configuration and present the equations of motion for the atoms driven to the dark state by the control and probe beams of light. In doing this we allow the two beams to have orbital angular momenta along the propagation axis $z$. In Sects. 4 and 5 we analyze the effective magnetic field and effective trapping potential in the case where at least one of the laser beams contains an OAM. We show that the spatial profile of the effective magnetic field can be controlled by applying proper control and probe beams. The concluding Sect. 6 summarizes the findings.

\section{General equations of the atomic motion}

\subsection{Translational motion for the multilevel atom}

Let us begin with a general treatment of the translational motion of an atom taking into account its internal degrees of freedom. The full atomic Hamiltonian is

$$
\hat{H}=\frac{\hat{\mathbf{p}}^{2}}{2 M}+\hat{H}_{0}(\mathbf{r})+\hat{V}(\mathbf{r}),
$$

where $\hat{\mathbf{p}} \equiv-\mathrm{i} \hbar \nabla$ is the momentum operator for an atom positioned at $\mathbf{r}$, and $M$ is the atomic mass. Here the Hamiltonian $\hat{H}_{0}(\mathbf{r})$ describes the electronic degrees of freedom of the atom, and $\hat{V}(\mathbf{r})$ represents an external trapping potential. Note that the Hamiltonian $\hat{H}_{0}(\mathbf{r})$ can also accommodate effects due to external light fields (if any). For fixed $\mathbf{r}$ the electronic Hamiltonian $\hat{H}_{0}(\mathbf{r})$ can be diagonalised to give a set of $N$ eigenvectors $\left|\chi_{n}(\mathbf{r})\right\rangle$ and eigenvalues $\varepsilon_{n}(\mathbf{r})$, with $n=$ $1,2, \ldots, N$. The full atomic wave function $\Phi$ is then expanded as

$$
|\Phi(\mathbf{r})\rangle=\sum_{n=1}^{N} \Psi_{n}(\mathbf{r})\left|\chi_{n}(\mathbf{r})\right\rangle,
$$

where a composite wave function $\Psi_{n}(\mathbf{r})$ describes the translational motion of the atom in the electronic state $n$.

Substituting Eq. (2) into the Schrödinger equation $\mathrm{i} \hbar \partial \Phi / \partial t=\hat{H} \Phi$, one arrives at a set of coupled equations for the components $\Psi_{n}$. Introducing a column $\Psi=\left(\Psi_{1}, \Psi_{2}, \ldots, \Psi_{N}\right)^{T}$, it is convenient to represent these equations in a matrix form:

$$
\mathrm{i} \hbar \frac{\partial}{\partial t} \Psi=\left[\frac{1}{2 M}(-\mathrm{i} \hbar \nabla-\mathbf{A})^{2}+U\right] \Psi,
$$

where $\mathbf{A}$ and $U$ are $N \times N$ matrices with the following elements:

$$
\begin{gathered}
\mathbf{A}_{n, m}=\mathrm{i} \hbar\left\langle\chi_{n} \mid \nabla \chi_{m}\right\rangle, \\
U_{n, m}=\varepsilon_{n}(\mathbf{x}) \delta_{n, m}+\left\langle\chi_{n}|\hat{V}(\mathbf{x})| \chi_{m}\right\rangle,
\end{gathered}
$$

i. e. the matrix $U$ includes contribution due to both the internal atomic energies and the external trapping potential.

\subsection{Adiabatic approximation}

Since the atomic internal motion is much faster than the external (translational) one, the difference in the atomic energies $U_{n, n}-U_{m, m}$ is normally much larger than the energies of non-adabatic coupling between these states. In such a situation the translational motion 
of atoms in different internal levels can be considered to be independent, leading to the adiabatic approximation. Specifically, let us suppose that a selected atomic level with $n=q$ is well separated from the remaining $N-1$ atomic levels. Neglecting transitions to the remaining states, Eq. (3) provides the adiabatic equation for the translational motion of the atom in the electronic state $q$ :

$$
\mathrm{i} \hbar \partial \Psi_{q} / \partial t=H_{\text {eff }} \Psi_{q},
$$

where

$$
H_{\text {eff }}=\frac{1}{2 M}\left(-\mathrm{i} \hbar \nabla-\mathbf{A}_{\text {eff }}\right)^{2}+U+\phi
$$

is the effective Hamiltonian of the atom in the electronic state $q$, with $\mathbf{A}_{\text {eff }} \equiv \mathbf{A}_{q, q}$ and $U \equiv U_{q, q}$ being defined by the above equations (4) and (5). An additional scalar potential $\phi$ appears due to the exclussion of a part of the electronic states in the effective equation of motion (7):

$$
\begin{aligned}
\phi & =\frac{1}{2 M} \sum_{l \neq q}^{N} \mathbf{A}_{q, l} \mathbf{A}_{l, q} \\
& =\frac{\hbar^{2}}{2 M}\left(\left\langle\nabla \chi_{q} \mid \nabla \chi_{q}\right\rangle+\left\langle\chi_{q} \mid \nabla \chi_{q}\right\rangle\left\langle\chi_{q} \mid \nabla \chi_{q}\right\rangle\right)
\end{aligned}
$$

The quantity $\mathbf{A}_{\text {eff }}$, called the Berry connection [17], represents an effective vector potential acting on the electrically neutral atoms. The effective vector potential $\mathbf{A}_{\text {eff }}$ appears due to the spatial dependence of the electronic state $\left|\chi_{q}\right\rangle$.

\section{Formulation}

\subsection{A system of atoms}

Let us now define the atomic system. We shall consider an ensemble of atoms characterized by two hyperfine ground levels 1 and 2, as well as an electronic excited level 3. The atoms interact with two resonant laser beams in the EIT configuration (see Fig. 1). The first beam (to be referred to as the control beam) drives the transition $|2\rangle \rightarrow|3\rangle$, whereas the second beam (the probe beam) is coupled with the transition $|1\rangle \rightarrow|3\rangle$, as shown in Fig. 1(a). The control laser has a frequency $\omega_{\mathrm{c}}$, a wave-vector $\mathbf{k}_{\mathrm{c}}$, and a Rabi frequency $\Omega_{\mathrm{c}}$. The probe field, on the other hand, is characterized by a central frequency $\omega_{\mathrm{p}}=c k_{\mathrm{p}}$, a wave vector $\mathrm{k}_{\mathrm{p}}$, and a Rabi frequency $\Omega_{\mathrm{p}}$. Of special interest is the case where the probe and control beams can carry OAM along the

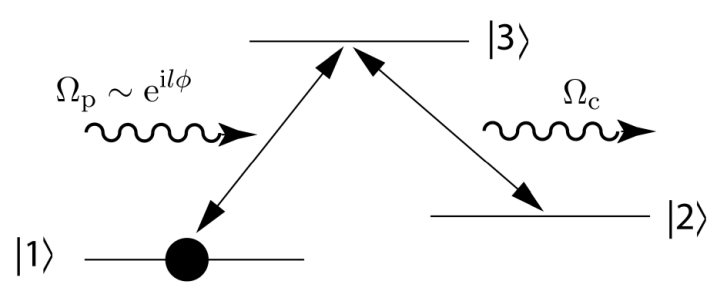

(a)

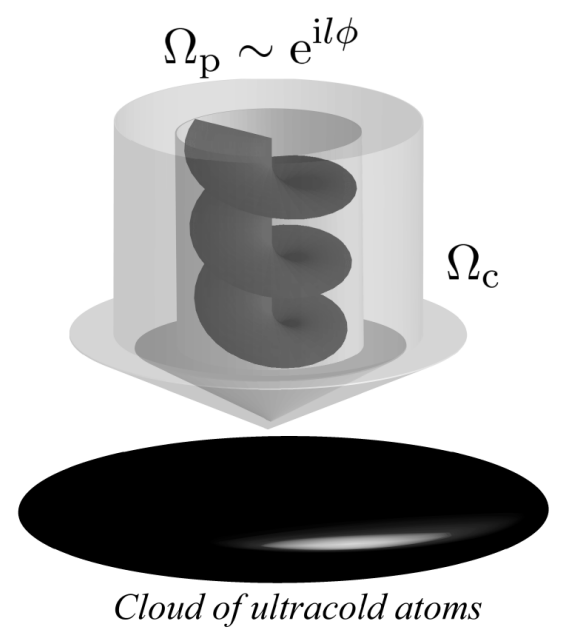

(b)

Fig. 1. (a) The level scheme for the $\Lambda$ type atoms interacting with the resonant probe beam $\Omega_{\mathrm{p}}$ and control beam $\Omega_{\mathrm{c}}$. (b) Schematic representation of the experimental setup with the two light beams incident on the cloud of atoms. The probe field is of the form $\Omega_{\mathrm{p}} \sim \mathrm{e}^{\mathrm{i} l \phi}$, where each probe photon carry an OAM $\hbar l$ along the propagation axis $z$.

propagation axis $z$. In that case, the spatial distribution of the beams is $[20,21]$

$$
\Omega_{\mathrm{p}}=\Omega_{\mathrm{p}}^{(0)} \mathrm{e}^{\mathrm{i}\left(k_{\mathrm{p}} z+l_{\mathrm{p}} \phi\right)}
$$

and

$$
\Omega_{\mathrm{c}}=\Omega_{\mathrm{c}}^{(0)} \mathrm{e}^{\mathrm{i}\left(k_{\mathrm{c}} z+l_{\mathrm{c}} \phi\right)},
$$

where $\Omega_{\mathrm{p}}^{(0)}$ and $\Omega_{\mathrm{c}}^{(0)}$ are slowly varying amplitudes for the probe and control fields, $\hbar l_{\mathrm{p}}$ and $\hbar l_{\mathrm{c}}$ are the corresponding orbital angular momenta per photon along the propagation axis $z$, and $\phi$ is the azimuthal angle.

\subsection{Hamiltonian for the electronic degrees of freedom of atom}

Adopting the rotating wave approximation, the Hamiltonian for the electronic degrees of freedom of an atom interacting with the control and probe fields in the rotating frame is

$$
\hat{H}_{0}(\mathbf{r})=\epsilon_{21}|2\rangle\left\langle 2\left|+\epsilon_{31}\right| 3\right\rangle\langle 3|
$$




$$
-\hbar\left(\Omega_{\mathrm{p}}|3\rangle\left\langle 1\left|+\Omega_{\mathrm{c}}\right| 3\right\rangle\langle 2|+\text { H. c. }\right),
$$

where $\epsilon_{21}=\hbar\left(\omega_{2}-\omega_{1}+\omega_{\mathrm{c}}-\omega_{\mathrm{p}}\right)$ and $\epsilon_{31}=$ $\hbar\left(\omega_{3}-\omega_{1}-\omega_{\mathrm{p}}\right)$ are, respectively, the energies of the detuning from the two- and single-photon resonances, with $\hbar \omega_{j}$ being the electronic energy of the atomic level $j$. Note that the spatial dependence of the Hamiltonian $\hat{H}_{0}(\mathbf{r})$ emerges through the spatial depandence of the Rabi frequencies $\Omega_{\mathrm{p}} \equiv \Omega_{\mathrm{p}}(\mathbf{r})$ and $\Omega_{\mathrm{c}} \equiv \Omega_{\mathrm{c}}(\mathbf{r})$.

Neglecting the two-photon detuning, the Hamiltonian (11) has the eigenstate

$$
|D\rangle=\frac{1}{\sqrt{1+|\zeta|^{2}}}(|1\rangle-\zeta|2\rangle)
$$

representing a coherent superposition of two hyperfine dark states. Here

$$
\zeta=\frac{\Omega_{\mathrm{p}}}{\Omega_{\mathrm{c}}}
$$

is the ratio of the amplitudes of the probe and control fields.

The state $|D\rangle$ is known as the dark state [22-25]. It is characterized by zero eigenenergy: $\hat{H}_{0}(\mathbf{r})|D\rangle=0$. Since the dark state has no contribution by the excited electronic state $|3\rangle$, it is immune from the spontaneous emission. We shall be interested in a situation where the atoms are driven to their dark states. If an atom is in the dark state $|D\rangle$, the resonant control and probe beams induce the absorption paths $|2\rangle \rightarrow|3\rangle$ and $|1\rangle \rightarrow|3\rangle$, which interfere destructively, resulting in the electromagnetically induced transparency [22-25]. In such a situation, the transitions to the upper atomic level 3 are suppressed. That's why the dark state has no contribution by the excited electronic state $|3\rangle$.

\subsection{Effective Hamiltonian for the dark-state atoms}

Suppose that the control and probe fields are tuned to the two-photon resonance: $\epsilon_{21}=0$. The remaining two-photon mismatch (if any) can be accommodated within the trapping potential

$$
\hat{V}(\mathbf{r})=V_{1}(\mathbf{r})|1\rangle\left\langle 1\left|+V_{2}(\mathbf{r})\right| 2\right\rangle\left\langle 2\left|+V_{3}(\mathbf{r})\right| 3\right\rangle\langle 3|,
$$

where $V_{j}(\mathbf{r})$ are the trapping potentials for an atom in the electronic state $j$, with $j=1,2,3$.

According to the treatment presented in the previous section, the dynamics of the dark-state atoms is described by the following effective Hamiltonian (see Eq. (7)):

$$
\hat{H}_{\text {eff }}=\frac{1}{2 M}\left(-\mathrm{i} \hbar \nabla-\mathbf{A}_{\mathrm{eff}}\right)^{2}+V_{\mathrm{eff}},
$$

where

$$
\mathbf{A}_{\text {eff }}=\mathrm{i} \hbar\langle D \mid \nabla D\rangle
$$

and

$$
V_{\text {eff }}=U+\phi
$$

are the effective vector and trapping potentials, with

$$
U=\frac{V_{1}(\mathbf{r})+|\zeta|^{2} V_{2}(\mathbf{r})}{1+|\zeta|^{2}}
$$

$$
\phi=\frac{\hbar^{2}}{2 M}\left(\langle D \mid \nabla D\rangle^{2}+\langle\nabla D \mid \nabla D\rangle\right) .
$$

Since $V_{1}(\mathbf{r})$ and $V_{2}(\mathbf{r})$ are the trapping potentials for an atom in the electronic states 1 and $2, U$ represents the external trapping potential for an atom in the dark state.

In this way, the effective trapping potential $V_{\text {eff }}$ is composed of the external trapping potential $U$ and the geometric scalar potential $\phi$. The former $U$ is determined by the shape of the trapping potentials $V_{1}(\mathbf{r})$ and $V_{2}(\mathbf{r})$, as well as the intensity ratio $|\zeta|^{2}$. The latter geometric potential $\phi$ is determined exclussively by the spatial dependence of the dark state $|D\rangle$ emerging through the spatial dependence of the ratio of the Rabi frequencies $\zeta=\Omega_{\mathrm{p}} / \Omega_{\mathrm{c}}$. Note that the effective vector potential $\mathbf{A}_{\text {eff }}$ has a geometric nature as well, because it also originates from the spatial dependence of the dark state.

\subsection{Adiabatic condition}

The separation between the energies of the dark state and the remaining (bright) atomic states is characterized by the total Rabi frequency $\Omega=\sqrt{\Omega_{\mathrm{p}}^{2}+\Omega_{\mathrm{c}}^{2}}$. Assuming that the control and probe fields are tuned to the one- and two-photon resonances $\left(\epsilon_{31}, \epsilon_{21} \ll \hbar \Omega\right)$, the adiabatic approach holds if the non-diagonal matrix elements in Eq. (3) are much smaller than the total Rabi frequency $\Omega$. This leads to the following condition:

$$
F \ll \Omega,
$$

where the velocity-dependent term

$$
F=\frac{1}{1+|\zeta|^{2}}|\nabla \zeta \cdot \mathbf{v}|
$$

reflects the two-photon Doppler detuning. Note that the condition (20) does not accommodate effects due to the decay of the excited atoms. The dissipation effects can be included by replacing the energy of the one-photon detuning $\epsilon_{31}$ by $\epsilon_{31}-\mathrm{i} \hbar \gamma_{3}$, where $\gamma_{3}$ is the excited state 
decay rate. In such a situation the dark state can be shown to acquire a finite lifetime

$$
\tau_{D} \sim \gamma_{3}^{-1} \Omega^{2} / F^{2},
$$

which should be large compared to other characteristic times of the system.

The condition (20) implies that the inverse Rabi frequency $\Omega^{-1}$ should be smaller than the time an atom travels a characteristic length, over which the amplitude or the phase of the ratio $\zeta=\Omega_{\mathrm{p}} / \Omega_{\mathrm{c}}$ changes considerably. The latter length exceeds the optical wavelength, and the Rabi frequency can be of the order of $10^{7}$ to $10^{8} \mathrm{~s}^{-1}$ [26]. Consequently, the adiabatic condition (20) should still hold for atomic velocities of the order of tens of metres per second, i. e., up to extremely large velocities in the context of ultracold atomic gases. The allowed atomic velocities become lower if the spontaneous decay of the excited atoms is taken into account. According to Eq. (22), the atomic dark state accquires then a finite lifetime $\tau_{D}$ which equals to $\gamma_{3}^{-1}$ times the ratio $\Omega^{2} / F^{2}$. The atomic decay rate $\gamma_{3}$ is typically of the order of $10^{7} \mathrm{~s}^{-1}$. Therefore, in order to achieve long-lived dark states the atomic speed should not be too large. For instance, if the atomic velocities are of the order of a centimeter per second (a typical speed of sound in an atomic BEC), the atoms should survive in their dark states up to a few seconds. This is comparable to the typical lifetime of an atomic BEC.

\section{Analysis of the effective vector and trapping potentials}

Using the expression (12) for the dark state, Eq. (16) for the effective vector potential takes the form

$$
\mathbf{A}_{\mathrm{eff}}=\mathrm{i} \hbar \frac{\zeta^{*} \nabla \zeta-\zeta \nabla \zeta^{*}}{2\left(1+|\zeta|^{2}\right)}
$$

Then the effective magnetic field reads:

$$
\mathbf{B}_{\mathrm{eff}}=\nabla \times \mathbf{A}_{\mathrm{eff}}=\mathrm{i} \hbar \frac{\nabla \zeta^{*} \times \nabla \zeta}{\left(1+|\zeta|^{2}\right)^{2}},
$$

and the geometric scalar potential is

$$
\phi=\frac{\hbar^{2}}{2 M} \frac{\nabla \zeta^{*} \nabla \zeta}{\left(1+|\zeta|^{2}\right)^{2}} .
$$

\subsection{Representation in terms of the amplitude and phase}

Let us to express the ratio of Rabi frequencies $\zeta$ in terms of amplitude and phase:

$$
\zeta=\frac{\Omega_{\mathrm{p}}}{\Omega_{\mathrm{c}}}=|\zeta| \mathrm{e}^{\mathrm{i} S} .
$$

The effective vector potential, the effective magnetic field, and the effective scalar potential are then

$$
\begin{aligned}
\mathbf{A}_{\mathrm{eff}} & =-\hbar \frac{|\zeta|^{2}}{1+|\zeta|^{2}} \nabla S, \\
\mathbf{B}_{\mathrm{eff}} & =\hbar \frac{\nabla S \times \nabla|\zeta|^{2}}{\left(1+|\zeta|^{2}\right)^{2}}, \\
\phi & =\frac{\hbar^{2}}{2 M} \frac{(\nabla|\zeta|)^{2}+|\zeta|^{2}(\nabla S)^{2}}{\left(1+|\zeta|^{2}\right)^{2}} .
\end{aligned}
$$

\subsection{Representation in terms of the mixing angle}

It is convenient to introduce the mixing angle $\alpha$ via the following relationships:

$$
\sin \alpha=\frac{1}{\sqrt{1+|\zeta|^{2}}}, \quad \cos \alpha=\frac{|\zeta|}{\sqrt{1+|\zeta|^{2}}} .
$$

Specifically, if $|\zeta|$ is much larger than unity, then $\alpha \approx$ $1 /|\zeta|$. On the other hand, if $|\zeta|$ is much smaller than unity, then $\alpha \approx \pi / 2-|\zeta|$.

The dark state can now be represented as

$$
|D\rangle=\sin \alpha|1\rangle-\cos \alpha \mathrm{e}^{\mathrm{i} S}|2\rangle .
$$

The effective vector and scalar potentials can also be rewritten in terms of the mixing angle:

$$
\mathbf{A}_{\mathrm{eff}}=-\hbar \cos ^{2} \alpha \nabla S=-\frac{\hbar}{2}(1+\cos (2 \alpha)) \nabla S
$$

and

$$
\begin{aligned}
\phi & =\frac{\hbar^{2}}{2 M}\left[\left(\frac{1}{2} \sin (2 \alpha) \nabla S\right)^{2}+(\nabla \alpha)^{2}\right] \\
& =\frac{\hbar^{2}}{8 M}\left[\left(1-\cos ^{2}(2 \alpha)\right)(\nabla S)^{2}+\frac{(\nabla \cos (2 \alpha))^{2}}{1-\cos ^{2}(2 \alpha)}\right] .
\end{aligned}
$$

i. e., both potentials can be expressed through the quantity

$$
\cos (2 \alpha)=\frac{|\zeta|^{2}-1}{|\zeta|^{2}+1}
$$

The same applies to the effective magnetic field:

$$
\mathbf{B}_{\text {eff }}=\nabla \times \mathbf{A}_{\text {eff }}=\frac{\hbar}{2} \nabla S \times \nabla \cos (2 \alpha) .
$$




\subsection{Co-propagating control and probe beams with $O A M$}

If the co-propagating probe and control fields carry OAM, their amplitudes $\Omega_{\mathrm{p}}$ and $\Omega_{\mathrm{c}}$ are given by Eqs. (9), (10). The phase of the ratio $\zeta=\Omega_{\mathrm{p}} / \Omega_{\mathrm{c}}$ then reads

$$
S=l \phi,
$$

where $l=l_{\mathrm{p}}-l_{\mathrm{c}}$. Note that although both the control and probe fields are generally allowed to have non-zero OAM by Eqs. (9), (10), it is desirable for the OAM to be zero for one of these beams. In fact, if both $l_{\mathrm{p}}$ and $l_{\mathrm{c}}$ were non-zero, the amplitudes $\Omega_{\mathrm{p}}$ and $\Omega_{\mathrm{c}}$ should simultaneously go to zero along the $z$ axis. In such a situation the total Rabi frequency $\Omega=\sqrt{\Omega_{\mathrm{p}}^{2}+\Omega_{\mathrm{c}}^{2}}$ would also vanish, leading to the violation of the adiabatic condition (20) along the $z$ axis.

Substituting Eq. (36) into Eqs. (32), (34), and (35), one has

$$
\begin{gathered}
\mathbf{A}_{\mathrm{eff}}=-\hbar \cos ^{2} \alpha \frac{l}{\rho} \mathbf{e}_{\varphi} \\
\phi=\frac{\hbar^{2}}{2 M}\left[\left(\frac{1}{2} \sin (2 \alpha) \frac{l}{\rho}\right)^{2}+(\nabla \alpha)^{2}\right],
\end{gathered}
$$

and

$$
\mathbf{B}_{\text {eff }}=\frac{\hbar}{2} \frac{l}{\rho} \mathbf{e}_{\varphi} \times \nabla \cos (2 \alpha),
$$

where $\mathbf{e}_{\varphi}$ is the unit vector in the azimuthal direction, and $\rho$ is the cylindrical radius.

In what follows the intensity ratio $|\zeta|^{2}$ is considered to depend on the cylindrical radius $\rho$ only. In that case the effective scalar potential and magnetic field reduce to

$$
\phi=\frac{\hbar^{2}}{2 M}\left[\left(\frac{1}{2} \sin (2 \alpha) \frac{l}{\rho}\right)^{2}+\left(\frac{\partial \alpha}{\partial \rho}\right)^{2}\right]
$$

and

$$
\mathbf{B}_{\text {eff }}=-\frac{\hbar}{2} \frac{l}{\rho} \frac{\partial}{\partial \rho} \cos (2 \alpha) \mathbf{e}_{z} .
$$

Consequently, the effective magnetic field is directed along the $z$ axis.

\subsection{Magnetic flux}

Suppose the probe beam has an OAM $\left(l_{\mathrm{p}} \neq 0\right)$ and the control beam has not $\left(l_{\mathrm{c}}=0\right)$. In this case the intensity of the probe beam (and hence the ratio $|\zeta|^{2}=$ $\left.\left|\Omega_{\mathrm{p}} / \Omega_{\mathrm{c}}\right|^{2}\right)$ goes to zero as $\rho \rightarrow 0$. If the intensity of the control field changes slowly within an atomic cloud, the $\rho$-dependence of the ratio $|\zeta|$ is determined by the probe beam only.

The effective magnetic flux through a circle of the radius $\rho_{0}$ is now given by

$$
\Phi=\oint \mathbf{A}_{\text {eff }} \mathrm{d} \mathbf{l}=-2 \pi \hbar \frac{l\left|\zeta_{0}\right|^{2}}{1+\left|\zeta_{0}\right|^{2}},
$$

where $2 \pi \hbar$ is the Dirac flux quantum, and $\left|\zeta_{0}\right|^{2}$ is the intensity ratio at the radius $\rho=\rho_{0}$. The flux $\Phi$ reaches its maximum of $2 \pi \hbar l$ if the ratio $\left|\zeta_{0}\right|^{2} \gg 1$, i.e. if the intensity of the probe field exceeds the control field at the selected radius $\rho_{0}$. Since the winding number of light beams can currently be as large as several hundred, it is possible to induce a substantial flux $\Phi$ in the atomic cloud. This might enable us to study phenomena related to filled Landau levels with a large number of atoms in the quantum gases.

\section{Specific cases}

\subsection{The polynomial case}

If we take

$$
|\zeta|=a \rho+b \rho^{2},
$$

then

$$
\cos (2 \alpha)=\frac{\left(a \rho+b \rho^{2}\right)^{2}-1}{\left(a \rho+b \rho^{2}\right)^{2}+1} .
$$

Consequently, one has

$$
\begin{aligned}
\mathbf{A}_{\mathrm{eff}}= & -\hbar l \frac{\rho(a+b \rho)^{2}}{1+\rho^{2}(a+b \rho)^{2}} \mathbf{e}_{\varphi}, \\
\phi= & \frac{\hbar^{2}}{2 M} \\
& \times \frac{\left(l^{2}+1\right) a^{2}+2\left(l^{2}+2\right) a b \rho+\left(l^{2}+4\right) b^{2} \rho^{2}}{\left(1+\rho^{2}(a+b \rho)^{2}\right)^{2}} .
\end{aligned}
$$

In this case the effective magnetic field

$$
\mathbf{B}_{\text {eff }}=-\hbar l \frac{2(a+b \rho)(a+2 b \rho)}{\left(1+\rho^{2}(a+b \rho)^{2}\right)^{2}} \mathbf{e}_{z}
$$

exhibits a radial dependence.

\subsection{Bessel beam}

Suppose the probe field represents a Bessel beam and the Rabi frequency of the control beam is almost constant within an atomic cloud. In such a case we have

$$
\zeta=b J_{l}(a \rho) \mathrm{e}^{\mathrm{i} l \varphi},
$$


where $b$ is a dimensionless constant determining the relative strength of the probe field. Then the effective scalar vector and scalar potentials are

$$
\begin{aligned}
\mathbf{A}_{\text {eff }}= & -\hbar \frac{b^{2} J_{l}(a \rho)^{2}}{1+b^{2} J_{l}(a \rho)^{2}} \frac{l}{\rho} \mathbf{e}_{\varphi}, \\
\phi= & \frac{\hbar^{2} b^{2}}{2 M} \\
& \times \frac{4 l^{2} J_{l}(a \rho)^{2}+a^{2} \rho^{2}\left(J_{l-1}(a \rho)-J_{l+1}(a \rho)\right)^{2}}{4 \rho^{2}\left(1+b^{2} J_{l}(a \rho)^{2}\right)^{2}} .
\end{aligned}
$$

In this case the effective magnetic field

$$
\begin{aligned}
\mathbf{B}_{\text {eff }}= & -\hbar \frac{a b^{2} l}{\rho} \\
& \times \frac{J_{l}(a \rho)\left(J_{l-1}(a \rho)-J_{l+1}(a \rho)\right)}{\left(1+b^{2} J_{l}(a \rho)^{2}\right)^{2}} \mathbf{e}_{z}
\end{aligned}
$$

also exhibits a radial dependence. Furthermore, from Eq. (50) it follows that the sign of the effective magnetic field alternates, i. e., the regions with the effective magnetic field aligned along $z$ axis are replaced by the regions in which the effective magnetic field is directed opposite to the $z$ axis and vice versa.

Next we shall examine situations where the effective magnetic field appears to be constant.

\subsection{Constant effective magnetic field}

If we choose

$$
|\zeta|^{2}=\frac{\left(\rho / \rho_{\max }\right)^{2}}{1-\left(\rho / \rho_{\max }\right)^{2}}
$$

the effective vector potential, Eq. (37), takes the form

$$
\mathbf{A}_{\text {eff }}=-\hbar l \rho \rho_{\max }^{-2} \mathbf{e}_{\phi} .
$$

Consequently, we arrive at a constant effective magnetic field

$$
\mathbf{B}_{\text {eff }}=-2 \hbar l \rho_{\max }^{-2} \mathbf{e}_{z}
$$

with the corresponding cyclotron frequency $\omega_{\mathrm{c}}=$ $\hbar 2 l /\left(M \rho_{\max }^{2}\right)$ and the magnetic length $l_{B}=$ $\sqrt{\hbar /\left(M \omega_{\mathrm{c}}\right)}=\rho_{\max } / \sqrt{2 l}$. The scalar potential is now given by

$$
\phi=\frac{\hbar^{2}}{2 M} \frac{1}{\rho_{\max }^{2}}\left(l^{2} d+1 / d\right)
$$

where $d=1-\left(\rho / \rho_{\max }\right)^{2}$. For $\rho \rightarrow \rho_{\max }$ the intensity ratio $|\zeta|^{2}$ goes to infinity, so the equations (51)-(54) have a meaning only for distances smaller than $\rho_{\max }$.

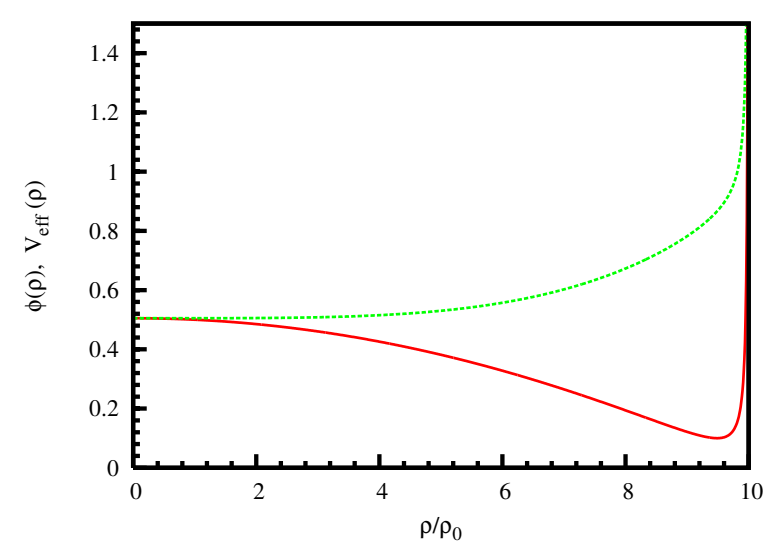

Fig. 2. Geometric scalar potential $\phi$ (solid line) and the effective trapping potential $V_{\text {eff }}$ (dashed line) for the case where the effective magnetic field $B_{\text {eff }}$ is constant. The trapping potential $V_{1}$ is given by Eq. (55) to compensate the quadratic term in $\phi$. The trapping potential for the atoms in the second hyperfine ground state is chosen to be $V_{2}=\kappa V_{1}$, with $\kappa=9 / 5$. The other constants are $M=1, \hbar=1, l=10, \rho_{0}=1$, and $\rho_{\max }=10$.

Therefore, Eq. (51) can model an actual intensity distribution of the control and probe beams only up to a certain radius $\rho_{0}$ which is smaller than $\rho_{\text {max }}$. When the radius $\rho_{0}$ is close to $\rho_{\max }$, the effective magnetic flux approaches its maximum value of $2 \pi \hbar l$.

The appearing scalar potential $\phi$ can be compensated by the trapping potential. If

$$
V_{1}(\mathbf{r})=\frac{\hbar^{2}}{2 M \rho_{\max }^{2}}\left(l^{2}-1\right)\left(\rho / \rho_{\max }\right)^{2}
$$

and $V_{2}(\mathbf{r})=\kappa V_{1}(\mathbf{r})$, the external trapping potential $U$ given by Eq. (18) compensates the quadratic term in Eq. (54). Assuming $\kappa=1$, the overall effective trapping potential $V_{\text {eff }}=U+\phi$ is flat almost up to the maximum radius $\rho=\rho_{\max }$.

Figure 2 shows the geometric scalar potential $\phi$ and the effective trapping potential $V_{\text {eff }}$ for the situation where $\kappa=9 / 5$. One can see that the effective trapping potential $V_{\text {eff }}$ is almost flat in the centre and increases with increasing radius $\rho$, despite the fact that the geometric scalar potential $\phi$ decreases and has a minimum near $\rho_{\max }$.

\subsection{Constant effective magnetic field for ring geometry}

In the previous subsection we have analyzed the constant effective field in the case where the atomic motion is restricted to the distances where $\rho<\rho_{\max }$. Here we shall consider the situation where the atomic motion is restricted additionally from below, i.e. $\rho>\rho_{\max }$. In 


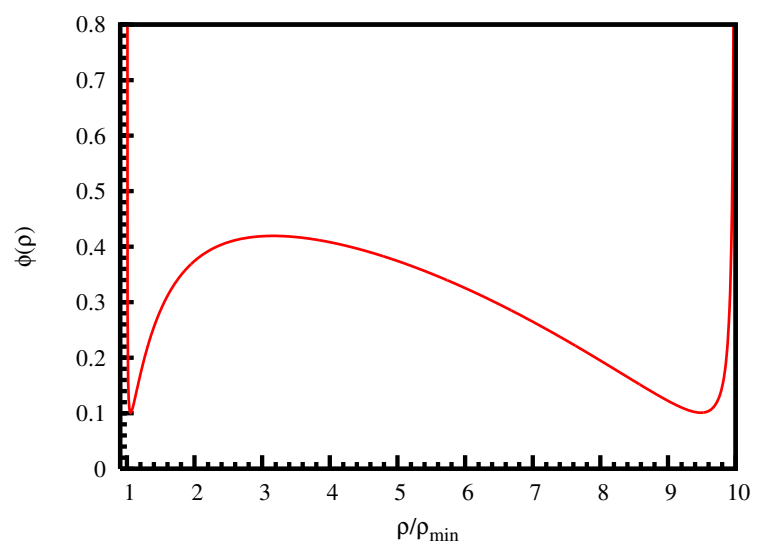

Fig. 3. Geometric scalar potential $\phi$ for the case of constant effective magnetic field $B_{\text {eff }}$ in the ring geometry. The constants are $M=1, \hbar=1, l=10, \rho_{\min }=1$, and $\rho_{\max }=10$.

such a case the constant effective magnetic field is obtained in the case where

$$
|\zeta|^{2}=\frac{\rho^{2}-\rho_{\min }^{2}}{\rho_{\max }^{2}-\rho^{2}} .
$$

The effective vector potential then takes the form

$$
\mathbf{A}_{\mathrm{eff}}=-\hbar \frac{\rho^{2}-\rho_{\min }^{2}}{\rho_{\max }^{2}-\rho_{\min }^{2}} \frac{l}{\rho} \mathbf{e}_{\varphi},
$$

giving the following magnetic field strength

$$
\mathbf{B}_{\text {eff }}=-\frac{2 \hbar l}{\rho_{\max }^{2}-\rho_{\min }^{2}} \mathbf{e}_{z} .
$$

The magnetic field strength is seen to increase with increasing $\rho_{\text {min }}$. Finally, the scalar potential is given by

$$
\begin{aligned}
\phi= & \frac{\hbar^{2}}{2 M}\left(\frac{l^{2}}{\rho^{2}} \frac{\left(\rho_{\max }^{2}-\rho^{2}\right)\left(\rho^{2}-\rho_{\min }^{2}\right)}{\left(\rho_{\max }^{2}-\rho_{\min }^{2}\right)^{2}}\right. \\
& \left.+\frac{\rho^{2}}{\left(\rho_{\max }^{2}-\rho^{2}\right)\left(\rho^{2}-\rho_{\min }^{2}\right)}\right) .
\end{aligned}
$$

The potential $\phi$ has singuliarities both at $\rho=\rho_{\text {min }}$ and $\rho=\rho_{\max }$, as illustrated in Fig. 3 .

\section{Conclusions}

We have studied the influence of two beams of light with orbital angular momenta on a degenerate gas of electrically neutral atoms (fermions or bosons). We have derived an equation of motion for atoms driven to a dark state. The equation contains a vector potential type interaction as well as an effective trapping potential. We have analyzed the effective vector and trapping potentials in the case where at least one of the light beams contains an OAM. We have shown how to generate a constant effective magnetic field both in the disc and ring geometries of the atomic trap. Furthermore, we have explored a couple of examples of the effective magnetic field exhibiting a radial distance dependence. We have demonstrated that the effective magnetic field can be concentrated in the area where the effective trapping potential holds the atoms. In the case of a homogeneous effective magnetic field it is important to realize that the corresponding cyclotron frequencies and magnetic lengths can be similar to typical trap frequencies and oscillator lengths used when trapping cold atoms in $\mathrm{BEC}$ and degenerate fermion gases. This will require a high OAM for the light which is also readily available with present technology.

Our proposed method of creating the effective magnetic field has several advantages compared to a rotating system where only a constant magnetic field is created [9-11]. In our method the magnetic field is shaped and controlled by choosing the proper control and probe beams. Furthermore stirring an ultracold cloud of atoms in a controlled manner is a rather demanding task, whereas an optically induced vector potential is expected to be highly controllable.

An effective magnetic field in an ultracold quantum gas gives rise to some remarkable scenarios. To experimentally verify the presence of an effective magnetic field one could for instance study the elementary excitations of the trapped cloud of atoms. This is an accurate tool to measure the properties of Bose-Einstein condensates or trapped Fermi gases. There are indeed an abundance of more extreme situations where the effective magnetic field plays a crucial role. The theory presented here has already been applied analyzing the de Haas-van Alphen effect in a gas of electrically neutral atoms [15]. It can also be applied to other intriguing phenomena which intrinsically depend on the magnetic field. For instance, the quantum Hall effect can now be studied using a cold gas of electrically neutral atomic fermions [27]. In addition, if the collisional interaction between the atoms is taken into account, we can study the magnetic properties of a superfluid atomic Fermi gas [28]. Recent advances in spatial light modulator technology enables us to consider rather exotic light beams [29]. This will allow us to study the effect of different forms of vector potentials in quantum gases. Finally, the combined dynamical system of light and matter [30] could give an important insight into gauge theories in general. 


\section{Acknowledgements}

This work was supported by the Royal Society of Edinburgh, the Alexander von Humboldt foundation and the Marie Curie Training Site at the University of Kaiserslautern. Helpful discussions with M. Fleischhauer are gratefully acknowledged.

\section{References}

[1] K.B. Davis, M.-O. Mewes, M.R. Andrews, N.J. van Druten, D.S. Durfee, D.M. Kurn, and W. Ketterle, Phys. Rev. Lett. 75, 3969 (1995).

[2] C.C. Bradley, C.A. Sackett, J.J. Tollett, and R.G. Hulet, Phys. Rev. Lett. 75, 1687 (1995).

[3] F. Dalfovo, S. Giorgini, L. Pitaevskii, and S. Stringari, Rev. Mod. Phys. 71, 463 (1999).

[4] L. Pitaevskii and S. Stringari, Bose-Einstein Condensation (Clarendon Press, Oxford, 2003).

[5] B. DeMarco and D. Jin, Science 285, 1703 (1999).

[6] F. Schreck, L. Khaykovich, K.L. Corwin, G. Ferrari, T. Bourdel, J. Cubizolles, and C. Salomon, Phys. Rev. Lett. 87, 080403 (2001).

[7] Z. Hadzibabic, S. Gupta, C.A. Stan, C.H. Schunck, M.W. Zwierlein, K. Dieckmann, and W. Ketterle, Phys. Rev. Lett. 91, 160401 (2003).

[8] D. Jaksch, C. Bruder, J.I. Cirac, C.W. Gardiner, and P. Zoller, Phys. Rev. Lett. 81, 3108 (1998).

[9] V. Bretin, S. Stock, Y. Seurin, and J. Dalibard, Phys. Rev. Lett. 92, 050403 (2004).

[10] V. Schweikhard, I. Coddington, P. Engels, V.P. Mogendorff, and E.A. Cornell, Phys. Rev. Lett. 92, 040404 (2004).
[11] M.A. Baranov, K. Osterloh, and M. Lewenstein, cond-mat / 0404329.

[12] D. Jaksch and P. Zoller, New J. Phys. 5, 56 (2003).

[13] E.J. Mueller, Phys. Rev. A 70, 041603(R) (2004).

[14] A. Sørensen, E. Demler, and M. Lukin, condmat/ 0405079 .

[15] G. Juzeliūnas and P. Öhberg, Phys. Rev. Lett. 93, 033602 (2004).

[16] G. Juzeliūnas, P. Öhberg, J. Ruseckas, and A. Klein, Phys. Rev. A 71, 053614 (2005).

[17] R. Jackiw, At. Mol. Phys. 21, 71 (1988).

[18] C.-P. Sun and M.-L. Ge, Phys. Rev. D 41, 1349 (1990).

[19] R. Dum and M. Olshanii, Phys. Rev. Lett. 76, 1788 (1996).

[20] L. Allen, M. Padgett, and M. Babiker, Prog. Opt. 39, 291 (1999).

[21] L. Allen, S.M. Barnett, and M.J. Padgett, Optical Angular Momentum (Institute of Physics, Bristol, 2003).

[22] E. Arimondo, Prog. Opt. 35, 259 (1996).

[23] S.E. Harris, Phys. Today 50, 36 (1997).

[24] A.B. Matsko, O. Kocharovskaja, Y. Rostovtsev, G.R. Welch, A.S. Zibrov, and M.O. Scully, Adv. At. Mol. Opt. Phys. 46, 191 (2001).

[25] M.D. Lukin, Rev. Mod. Phys. 75, 457 (2003).

[26] L.V. Hau, S.E. Harris, Z. Dutton, and C. Behrooz, Nature 397, 594 (1999).

[27] P. Öhberg, G. Juzeliūnas, J. Ruseckas, and M. Fleischhauer, to be submitted to Phys. Rev. A.

[28] C.A. Regal, M. Greiner, and D.S. Jin, Phys. Rev. Lett. 92, 040403 (2004).

[29] D. McGloin, G. Spalding, H. Melville, W. Sibbett, and K. Dholakia, Opt. Express 11, 158 (2003).

[30] P. Öhberg, Phys. Rev. A 66, 021603(R) (2002).

\title{
EFEKTYVUSIS MAGNETINIS LAUKAS LABAI ŠALTOSE ATOMU DUJOSE
}

\author{
G. Juzeliūnas ${ }^{\mathrm{a}}$, J. Ruseckas ${ }^{\mathrm{a}}$, P. Öhberg ${ }^{\mathrm{b}}$ \\ ${ }^{\text {a } T e o r i n e s ~ f i z i k o s ~ i r ~ a s t r o n o m i j o s ~ i n s t i t u t a s, ~ V i l n i u s, ~ L i e t u v a ~}$ \\ b Stratklaido universitetas, Glazgas, Škotija
}

\begin{abstract}
Santrauka
Nagrinėtas dvieju rezonansiniu šviesos pluoštu poveikis šaltu atomu dujoms. Ištirtas atvejis, kai bent vienas tų pluoštu turi nenulinę orbitinio judesio kiekio momento projekciją išilgai sklidimo krypties, o šviesai sąveikaujant su atomais, susidaro elektromagnetiškai sukeltas praskaidrèjimas. Parodyta, kad tuomet šviesos pluoštų poveikis atomų masių centro judejimui yra panašus i magnetinio lauko poveiki elektringoms dalelèms. Remiantis mikroskopiniu lètai sklindančios šviesos bei atomu aprašymu, buvo išvesta atomų masių centro judejjimo kvantinè lygtis, kurioje atsiranda efektyvusis vektorinis potencialas $\mathbf{A}_{\text {eff }}$, imituojantis magnetinį lauką. Efektyviojo magnetinio lauko indukcijos $\mathbf{B}_{\text {eff }}$ erdvini
\end{abstract}

pasiskirstymą galima reikiamai suformuoti, pasirenkant atitinkamos formos šviesos pluoštus. Išnagrinèta keletas šviesos pluoštų pavidalų. Rastas pluoštu profilis, sukeliantis pastovu efektyvųji magnetini lauką disko ir žiedo formos atomų gaudyklèse. Efektyvaus magnetinio lauko sukūrimas yra svarbus klausimas, kadangi, kitaip negu elektronai metaluose, atomu Bose ir Einstein'o kondensatą ar išsigimusias Fermio dujas sudaro elektriškai neutralios dalelès (atomai), kurių neveikia tikras magnetinis laukas. Parodyta, kad efektyvusis magnetinis laukas gali būti pakankamai stiprus ir ji atitinkantis magnetinis ilgis gali gerokai viršyti bandinio plotị. Todèl atominèse dujose gali pasireikšti elektronu teorijoje žinomų magnetinių efektų analogai. 